\title{
An investigation of the mechanisms underlying the effects of viewing distance and stimulus length on attentional asymmetries during line bisection
}

\author{
Michael E. R. Nicholls ${ }^{1}$ • Emily Beckman ${ }^{1}$ • Owen Churches ${ }^{1}$
}

Published online: 5 May 2016

(C) The Psychonomic Society, Inc. 2016

\begin{abstract}
Clinical neglect patients overattend to stimuli on their right, whereas the general population overattend to the left (pseudoneglect). Both phenomena are affected by viewing distance, whereby the attentional biases are attenuated as the stimulus moves from near to far space. Both are also affected by stimulus length and reduce in strength, or even reverse (the crossover effect), as length decreases. To gain an insight into the cognitive/neural mechanisms that underlie the effects of viewing distance and stimulus length, in two experiments we examined the interaction between the variables. In Experiment 1 we asked university students $(n=20)$ to perform a horizontal landmark bisection task with lines presented at varying lengths $\left(1.2^{\circ}, 6.3^{\circ}\right.$, and $18.4^{\circ}$ of viewing angle) and distances (450 and $1,350 \mathrm{~mm}$ ). A crossover effect and pseudoneglect were observed for the short and the long lines, respectively. An effect of viewing distance was only observed for long lines. Experiment 2 was the same, except that the lines were rotated to form vertical lines. No crossover effect was observed for the short lines, but an upward bias was observed for the long lines. Once again, an effect of viewing distance was only apparent for the long lines. These results demonstrate that the crossover effect is not a general property of short lines and is specific to the horizontal dimension. Models of crossover therefore need to incorporate processes related to left-right asymmetries. The results also demonstrate that viewing distance only affects long lines, and that this happens irrespective of orientation. A model of viewing distance is discussed that incorporates a right hemisphere mechanism
\end{abstract}

Michael E. R. Nicholls

mike.nicholls@flinders.edu.au

School of Psychology, Flinders University, GPO Box 2100, Adelaide, SA 5001, Australia specialized for an interaction between the ventral and dorsal streams.

Keywords Horizontal · Vertical · Neglect · Pseudoneglect · Near $\cdot$ Far $\cdot$ Dorsal $\cdot$ Ventral

Spatial attention is not distributed evenly across the visual field, and asymmetries exist along the horizontal, vertical, and radial axes. Along the horizontal axis, the most dramatic example comes from clinical neglect patients with damage to the right parietal lobe, who ignore the left side of space and overattend to the right (Heilman, Watson, \& Valenstein, 1993). More subtle attentional asymmetries also occur in the general population. One such asymmetry, known as pseudoneglect (Bowers \& Heilman, 1980), appears to mirror many of the features of clinical neglect (Jewell \& McCourt, 2000). Pseudoneglect causes people to overestimate the leftward side of a stimulus as compared to the right. The leftward bias can be seen in tasks, such as line bisection, in which participants overestimate the relative length on the left (McCourt, 2001; McCourt \& Jewell, 1999; Thomas, Castine, Loetscher, \& Nicholls, 2015). A leftward overestimation is seen for a range of other discriminations, such as luminance discrimination (Nicholls, Bradshaw, \& Mattingley, 1999), for visual search (Nicholls et al., 2014), and even for the left/right mental representations of stimuli (Loftus \& Nicholls, 2012; Loftus, Nicholls, Mattingley, Chapman, \& Bradshaw, 2009).

Pseudoneglect is often explained with reference to models of attentional asymmetry generated by the left and right cerebral hemispheres (e.g., Heilman \& Van Den Abell, 1980). These models assume that the right hemisphere is specialized for tasks that engage spatial attention (see Fink et al., 2000). Therefore, when faced with a task such as line bisection, 
which requires spatial attention, the right hemisphere is more activated than the left. Kinsbourne (1993) proposed that differential levels of activation between the hemispheres cause a bias of attention toward the hemispace contralateral to the more activated hemisphere. As a result, the act of spatial processing during line bisection precipitates a bias of attention to the left hemispace, causing the features of the stimulus on that side to appear more salient. More recent models have proposed a revised activation-orientation model, in which pseudoneglect and neglect are explained in terms of asymmetric interhemispheric neural activation and connectivity (Siman-Tov et al., 2007). Neuroanatomical support for these models has come from a diffusion tensor-imaging study by Thiebaut de Schotten et al. (2011), which showed that individual differences in the strengths of pseudoneglect were related to asymmetries in the neural pathways connecting the ventral and dorsal attentional systems in the right hemisphere. Besides imbalances in attention, it has been proposed that cultural effects, such a reading, affect perceptual asymmetries (Chokron \& Imbert, 1993). Readers of English may therefore overestimate the left of a stimulus because of their left-to-right reading habits, as compared to readers of Hebrew, with a rightto-left reading direction. Although it appears that reading habits do affect perceptual asymmetries (Chokron \& Imbert, 1993), it also seems that the effect of reading direction is a moderator rather than a generator of pseudoneglect (Rinaldi, Di Luca, Henik, \& Girelli, 2014).

Viewing distance is known to be an important factor in the manifestation of both clinical neglect and pseudoneglect. For clinical patients, a dissociation is observed whereby symptoms of leftward neglect are strong for stimuli placed within reach (peripersonal space), and abate for stimuli placed outside of reach (extrapersonal space) (Aimola, Schindler, Simone, \& Venneri, 2012). A similar effect of distance is observed in the general population. For example, Longo and Lourenco (2006) presented lines at distances ranging between 300 and $1,200 \mathrm{~mm}$ and asked participants to bisect the lines using either a stick or a laser pointer. When the lines were bisected with a stick, which brought the stimuli "within reach" (see Iriki, Tanaka, \& Iwamura, 1996, for an animal model, and Berti \& Frassinetti, 2000, for a human model), a consistent leftward bisection was observed. Conversely, when the lines were bisected using a laser pointer, which allowed the lines to pass out of reach, a leftward bias was observed for the near lines, which turned into a rightward bisection bias for the far lines. Similar results have been reported by Gamberini, Seraglia, and Priftis (2008) for near and far stimuli in both a real and a virtual reality environment. Although a rightward bisection bias for lines in far space has also been reported by Nicholls, Jones, and Robertson (2016), it should be noted that sometimes the leftward attentional bias is only reduced in the far condition (Bjoertomt, Cowey, \& Walsh, 2002; Longo Trippier, Vagnoni, \& Lourenco, 2015). As an example, McCourt and Garlinghouse
(2000) presented prebisected lines at viewing distances of 450 and $900 \mathrm{~mm}$ and found a leftward bias for close lines, which was significantly reduced for far lines.

The effect of viewing distance on asymmetries in line bisection is commonly explained with reference to the relative activations of different neural systems that are specialized for the processing of near and far space (for a review, see Konen \& Kastner, 2008). Building upon findings of primate research by Rizzolatti, Matelli, and Pavesi (1983), Weiss et al. (2000) used regional cerebral blood flow to demonstrate the activation of brain regions associated with the dorsal (intraparietal sulcus) and ventral (medial temporal cortex) streams for the bisection of lines placed in near and far space, respectively. It is therefore possible that stimuli located in near space activate parietal/dorsal mechanisms, which are also implicated in the generation of pseudoneglect (Bjoertomt et al., 2002; Foxe, McCourt, \& Javitt, 2003; Waberski et al., 2008)—leading to a leftward bias. In contrast, stimuli located in far space activate temporal/ventral mechanisms, which are not so closely tied to the generation of pseudoneglect. Recent support for such a model has come from Longo et al. (2015). They measured electroencephalography (EEG) while participants carried out a landmark task in near and far space and found strong, typical activation of right parietal/occipital regions when the stimuli were located in near space, which diminished as the stimuli moved into far space. The effect of viewing distance on hemispheric asymmetry was especially pronounced between 240 and $400 \mathrm{~ms}$, suggesting a relatively late stage of processing. Longo et al. went on to suggest that the right ventral superior longitudinal fasciculus, identified by Thiebaut de Schotten et al. (2011) as a neural substrate of pseudoneglect, may be particularly important for the visuospatial processing of lines located in near space.

Stimulus length is another important factor that affects the degree and direction of both clinical neglect and pseudoneglect (see Jewell \& McCourt, 2000, for a review). As an example of this research, McCourt and Jewell (1999) asked participants to indicate whether prebisected lines (i.e., the landmark task) were transected to the left or right of true center. The lines varied in length from $1.48^{\circ}$ to $31.47^{\circ}$ of viewing angle (VA), in six steps. McCourt and Jewell found the expected leftward attentional bias for the longer lines, which was reduced as the lines became shorter. Finally, for the shortest line $\left(1.48^{\circ}\right)$, a reversal toward a rightward attentional bias was observed. This reversal, known as the crossover effect, was first noted in patients with spatial neglect, whereby the rightward bisection bias for longer lines crosses over to a leftward bisection bias for very short lines (Moonaghan \& Shillcock, 1998). A similar effect has been observed in the general population. In this case, however, the leftward bisection bias for longer lines crosses over to a rightward bias for very short lines (Rueckert, Deravanesian, Baboorian, Lacalamita, \& Repplinger, 2002). 
Benwell, Harvey, Gardner, and Thut (2013) suggested that pseudoneglect and the crossover effect both stem from attentional processes related to the activity of the hemispheres. For example, long lines are known to engage right hemisphere processing mechanisms more than short lines do (Benwell, Harvey, \& Thut, 2014). The additional right hemisphere activation caused by longer lines may therefore combine with the existing right hemisphere activity for the task to increase the leftward overestimation of the line. To support their theory, Benwell et al. (2013) independently manipulated line length and arousal during a line bisection task. Reductions in both line length and arousal are known to reduce leftward attentional biases - and possibly to lead to rightward biases instead (Benwell et al., 2013). Results have shown that line length and fatigue have additive effects, suggesting that the bisection asymmetries for short and long lines are governed by a common set of processes. A similar additive effect was observed by Benwell, Thut, Grant, and Harvey (2014) for the effect of ageing, which is also known to reduce right hemisphere activity.

To investigate the neural mechanisms that underlie the effect of length more closely, Benwell, Harvey, and Thut (2014) asked participants to make estimates of the relative lengths of the left and right sides of a prebisected line presented at a viewing distance of $1.0 \mathrm{~m}$, which were either short $\left(1^{\circ}\right.$ of VA) or long ( $15.3^{\circ}$ of VA). Significant leftward overestimation (pseudoneglect) was observed for the long lines, whereas the short lines produced a leftward bias that was not significantly different from zero (no bias). EEG recordings were made while participants carried out the task. Source localization showed that the effect of line length was associated with activity of the tempero-parietal junction in the right hemisphere, which is associated with the ventral attentional system. Benwell, Harvey, and Thut suggested that long lines selectively activate resources within the right hemisphere ventral network, whereas short lines do not activate these same areascausing a reduction of pseudoneglect. It is noteworthy that the EEG asymmetries arose between 100 and $200 \mathrm{~ms}$ - suggesting a relatively early stage of processing. The effect of line length therefore contrasts with the data reported by Longo et al. (2015), which showed late processing for the effect of viewing distance - suggesting a temporal dissociation in the effects of stimulus length and viewing distance on line bisection. Finally, although Benwell, Harvey, and Thut observed a dissociation between the ventral and dorsal attentional areas as a function of line length, it should be noted that this effect may be specific to the effect of line length. The implication that long lines, which generate stronger pseudoneglect, are associated with ventral-stream processing is not compatible with the larger literature pointing to a role of the right dorsal stream in the generation of pseudoneglect in near space (e.g., Bjoertomt et al., 2002; Foxe et al., 2003; Waberski et al., 2008; Weiss et al., 2000).
Important insights into the cognitive/neural mechanisms that underlie the effects of viewing distance and stimulus length can be gained by examining the interaction between the two variables. If an interaction is found, it suggests a dissociation in the mechanisms engaged by the task. One study to do this was carried out by Varnava, McCarthy, and Beaumont (2002). They asked participants to move a cursor along a horizontal line to indicate the perceived middle. The lines were presented at four viewing distances, ranging from 300 to $1,200 \mathrm{~mm}$. The lines ranged in length from $3.8^{\circ}$ to $14.9^{\circ}$ of VA. Finally, the cursor could start on the left or the right side. Varnava et al. found the expected effect of distance, whereby a leftward bias was found in near space, which changed to a rightward bias in far space. Surprisingly, they observed no effect of line length and no evidence of a crossover effect for the shortest line. The lack of a crossover effect may be related to the fact that the shortest stimulus was significantly longer than the stimuli used by Rueckert et al. (2002), which subtended approximately $1.4^{\circ}$ of VA. In addition, no interaction between viewing distance and line length emerged. There was, however, a three-way interaction - suggesting that dissociated neural systems are responsible for processing information in near and far space.

Heber, Siebertz, Wolter, Kuhlen, and Fimm (2010) also examined the interaction between stimulus length and viewing distance. Using a virtual-reality technique, stimuli were presented at a virtual viewing distance of 300 or $2,700 \mathrm{~mm}$ and ranged in length from $22.6^{\circ}$ to $46.6^{\circ}$ of VA. Participants were asked to carry out a greyscales task as well as a landmark task. For the horizontal trials, pseudoneglect was stronger for both tasks in near than in far space. Both tasks also showed the expected effect of length, which did not interact with viewing distance. They found no crossover effects - as might be expected for relatively long stimuli.

Although the studies by Varnava et al. (2002) and Heber et al. (2010) manipulated viewing distance and stimulus length within single experiments, neither found an interaction between viewing distance and stimulus length. Both studies therefore suggest that similar sets of cognitive/neural mechanisms are engaged by manipulations of both variables (notwithstanding the third-order interaction with scanning direction observed by Varnava et al., 2002). The failure to find an interaction, however, may have been related to the fact that a limited range of stimulus lengths were used. In particular, neither study used very short lines, which might have induced a crossover effect. In the present study, we sought to address this issue by using stimuli specifically designed to induce pseudoneglect and the crossover effect and to examine the effects of viewing distance on both phenomena. In the first experiment, we examined the effects of stimulus length and viewing distance for horizontally aligned lines. The second experiment generalized the experimental paradigm to the vertical dimension, in which attentional asymmetries are also 
known to exist (Drain \& Reuter-Lorenz, 1996; McCourt \& Olafson, 1997; Nicholls, Mattingley, Berberovic, Smith, \& Bradshaw, 2004). By applying the same paradigm across the two dimensions, we sought to gain a much better insight into the mechanisms that are affected by viewing distance and stimulus length.

\section{Experiment 1}

In the first experiment, we examined the effect of viewing distance (near [450 mm] and far [1,350 mm]) on bisection asymmetries in a horizontal landmark line bisection task, using (a) short, (b) medium, and (c) long lines.

(a) The short lines subtended $1.27^{\circ}$ of VA. Given that Rueckert et al. (2002) had observed a crossover effect for lines of approximately $1.4^{\circ} \mathrm{VA}$, a similar crossover effect was expected. How the response asymmetry would be affected by viewing distance was not certain, given that this condition had not been tested before. If an interaction were found, it would suggest that the ventral/ dorsal mechanisms purported to play a role in the effect of viewing distance (Bjoertomt et al., 2002) are specific to long lines.

(b) The medium lines subtended $6.34^{\circ}$ of VA. At this length, minimal pseudoneglect has been reported (McCourt \& Jewell, 1999). No response asymmetry or only a weak bias toward the left was therefore expected in this condition. How the data would be affected by viewing distance was once again uncertain - though an interaction would suggest a dissociation in the underlying mechanisms.

(c) The long lines subtended $18.43^{\circ}$ of VA. In the near viewing condition, an overestimation of the leftward features, consistent with the effect of pseudoneglect, was expected (McCourt, 2001; McCourt \& Jewell, 1999; Thomas et al., 2015). In the far condition, the leftward bias was expected to be either reversed to a rightward bias (Longo \& Lourenco, 2006; Nicholls et al., 2016) or extinguished (Bjoertomt et al., 2002; Longo et al., 2015; McCourt \& Garlinghouse, 2000).

\section{Method}

Participants Twenty university students (15 females, five males) participated in the experiment to receive course credit. The participants' ages ranged between 17 and 19 years $(M=$ 18.25 years), and all had normal or corrected-to-normal vision. Handedness was assessed using the FLANDERS handedness inventory (Nicholls, Thomas, Loetscher, \& Grimshaw, 2013), which gives scores ranging from -10 (completely lefthanded) to +10 (completely right-handed). The present experiment included participants who were all right-handed with scores ranging between +6 and $+10(M=+9.25)$. The study was approved by the Human Research Ethics Committee at Flinders University.

Apparatus Stimulus presentation was controlled with a PC running the E-Prime 2.0 software and was displayed on an LCD screen (Dell U3014) with a diagonal width of $750 \mathrm{~mm}$. The screen was run at a resolution of $2,560 \times 1,600$ pixels at $60 \mathrm{~Hz}$. Responses were recorded using the " $\mathrm{f}$ " and " $\mathrm{j}$ " keys of the computer's keyboard. A height-adjustable chinrest maintained participants' head position so that the center of the display panel was in line with their midsagittal plane at eye level at a distance of either 450 or $1,350 \mathrm{~mm}$.

Stimuli Prebisected horizontal lines were drawn in black against a white background. In the "near" condition, in which stimuli were viewed at a distance of $450 \mathrm{~mm}$, the lines were one pixel $\left(0.25 \mathrm{~mm}, 0.03^{\circ}\right.$ of VA) thick and 10,50 , or $150 \mathrm{~mm}$ long $\left(1.27^{\circ}, 6.34^{\circ}\right.$, or $18.43^{\circ}$ of VA). The ends of the lines were marked with a vertical stroke of the same width and $5 \mathrm{~mm}$ high $\left(0.63^{\circ}\right.$ of VA). The lines were transected with a vertical stroke of the same dimensions as the end markers (see Fig. 1). The transector was placed either in the exact horizontal middle of the line or 1 or $3 \mathrm{~mm}\left(0.12^{\circ}\right.$ or $\left.0.36^{\circ} \mathrm{VA}\right)$ to the left or right of the true middle (i.e., there were five different placements of the transector). To prevent participants from using a landmark on the screen to facilitate their judgments (e.g., a speck of dust on the screen), the horizontal positions of the stimuli were "jittered" by $10 \mathrm{~mm}\left(1.2^{\circ}\right.$ of VA) to the left or right of the true horizontal middle of the screen between trials.

In the "far" condition, stimuli were viewed at a distance of $1,350 \mathrm{~mm}$. The stimuli in this condition were exactly the same as those in the near condition, except that they were scaled up by a factor of 3 in both the vertical and horizontal dimensions. Thus, when viewed at the greater distance, they subtended exactly the same visual angle on the retina. Although this procedure did control for the relative size of the image as it fell on the retina and has successfully been used by Longo and Lourenco (2006), it should also be acknowledged that the absolute size of the image may also affect line bisection judgments in near and far space (Wilkinson \& Halligan, 2003).

Procedure The experiment was carried out in a well-lit room that contained a long table along which the monitor could be moved. Participants were seated at the end of the table with their head in a chinrest. The monitor was placed at eye level in line with participant's midsagittal axis, and was moved between blocks to create the near and far viewing conditions (see Fig. 1). Each trial began with the presentation of a prebisected line. Participants were asked to inspect the line and decide whether the left or right segment of the line appeared longer. Responses were made using a computer 


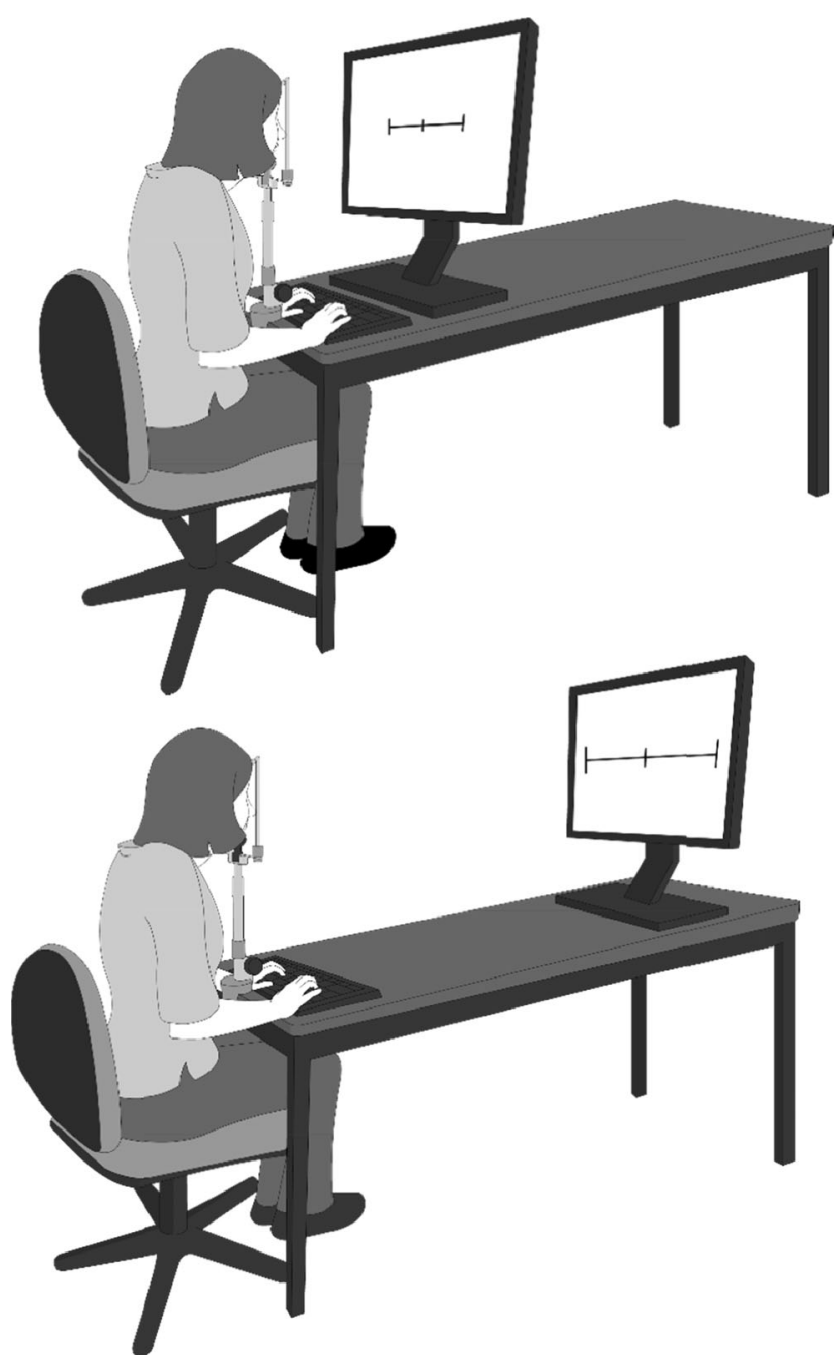

Fig. 1 Diagram showing the experimental setups in the near and far conditions. The screen and keyboard are aligned for the horizontal stimuli used in Experiment 1. Both the keyboard and screen were rotated $90^{\circ}$ for the vertical stimuli used in Experiment 2

keyboard on the table aligned with the participant's midsagittal axis. To indicate that the left or right segment appeared longer, participants pushed the " $\mathrm{f}$ " or the " $\mathrm{j}$ " key with the index finger of the left or the right hand, respectively. Because of the intuitive mapping between the stimulus and response positions, the hand of response was not varied between participants. Following a response the display was cleared, and a new trial was begun after a delay of $1.5 \mathrm{~s}$.

The near and far conditions were run as two separate blocks of trials, with order balanced between participants. Each block contained 150 trials and had a 3 (length: short $1.27^{\circ}$, medium $6.34^{\circ}$, long $18.43^{\circ}$ of VA) $\times 5$ (bisection point: $-0.36^{\circ},-0.12^{\circ}$, $0^{\circ},+0.12^{\circ},+0.36^{\circ}$ of VA) $\times 2$ (jitter: $-1.2^{\circ},+1.2^{\circ}$ ) factorial structure. Therefore, 30 unique stimuli were presented, with five repeats within each block. The order in which the different factorial combinations occurred within a block was randomized and balanced within the block. Participants completed one block of 12 practice trials prior to commencing the experimental trials.

\section{Results and discussion}

A measure of error was calculated by first removing the trials in which the transector was in the exact center of the linethat is, in which there was no correct response. The remaining errors were then summed and converted into a percentage of the total number of trials. The average overall error rate was $22.2 \%(S D=9.6)$. It is therefore clear that participants were attending to the task and were able to carry out the bisection judgments within an expected range of accuracy.

To gauge asymmetries in the estimation of length, a measure of response asymmetry was calculated by subtracting the number of "left longer" responses from the number of "right longer" responses. This difference score was then converted to a percentage of the total number of trials in that condition. Scores therefore ranged from -100 to +100 , with negative and positive scores reflecting overestimations of the left and right sides of the transector, respectively. The data were summed across the different transector and jitter positions - - leaving the factors of length and distance.

The data were analyzed using a series of one-sample $t$ tests to determine whether the response asymmetry was significantly different from zero in any of the six length/distance combinations. We observed a significant rightward bias for short lines in both the near $[t(19)=3.645, p=.002]$ and the $\operatorname{far}[t(19)=2.346, p=.030]$ conditions. This rightward bias is consistent with the crossover effect observed by Rueckert et al. (2002) for very short lines. For the medium lines $\left(6.34^{\circ}\right.$ of VA), there was no response asymmetry for either the near or the far condition. A similar null effect has been reported for lines with lengths around $5.90^{\circ}$ of VA by McCourt and Jewell (1999), and this may reflect an attenuation of pseudoneglect with decreases in length (notwithstanding the crossover effect). Finally, for the long lines, we found a significant leftward bias in the near condition $[t(19)=3.664, p=.002]$ —but not in the far condition. The leftward bias in the near condition is consistent with the effect of pseudoneglect (McCourt, 2001; McCourt \& Jewell, 1999; Thomas et al., 2015). The fact that pseudoneglect was present in the near but not the far condition most likely reflects the effect of viewing distance on pseudoneglect (Longo \& Lourenco, 2006; Nicholls et al., 2016). In this case, however, the asymmetry does not seem to have reversed to a rightward bias - but instead is in accord with reports of an attenuation of the leftward bias in far space (Bjoertomt et al., 2002; McCourt \& Garlinghouse, 2000). Longo et al. (2015) suggested that the failure to observe a reversal to a rightward bias in the far condition is related to the level of manual activation involved. For example, overt manual line bisections with a laser pointer (e.g., Longo \& Lourenco, 2006) may increase activation in the left 
hemisphere - producing a rightward bisection bias. In contrast, covert button presses (as in the present study) may produce less activation in the left hemisphere-resulting in a reduction of the leftward bisection bias. Figure 2 marks the conditions that were significantly different from zero with asterisks.

The response asymmetry data were analyzed with an analysis of variance (ANOVA) with Length (short, medium, long) and Distance (near, far) as within-participants factors. Effect size is represented by the partial eta-square value. We found a significant effect of length $\left[F(2,38)=12.951, p<.001, \eta_{\mathrm{p}}{ }^{2}=\right.$ .405]. Post-hoc analyses with a Bonferroni-corrected $p$ value of .016 revealed a significant difference between the short and medium lines $[t(19)=3.115, p=.006]$, as well as between the medium and long lines $[t(19)=3.088, p=.006]$. No effect of distance was apparent $\left[F(1,19)=1.195, p=.288, \eta_{\mathrm{p}}{ }^{2}=.059\right]$. There was, however, a significant interaction between length and distance $\left[F(1,19)=5.716, p=.007, \eta_{\mathrm{p}}{ }^{2}=.231\right]$ (see Fig. 2). Post-hoc analyses demonstrated that distance had no effect on the response asymmetries for short $[t(19)=0.024, p$ $=.981]$ and medium lines $[t(19)=1.450, p=.163]$. In contrast, for long lines, the leftward asymmetry was weaker in the far than in the near condition $[t(19)=2.391, p=.027]$ - though this effect was not significant according to the Bonferroni correction.

Of particular importance to the present study, the data showed an interaction between line length and distance. Long lines produced the expected effect of distance, whereby pseudoneglect was present for the near viewing condition, but absent for the far condition (Bjoertomt et al., 2002; McCourt $\&$ Garlinghouse, 2000). The effect of viewing distance is consistent with differential activation of the ventral and dorsal visual pathways by far and near objects, respectively (Bjoertomt et al., 2002) and with the idea that the dorsal

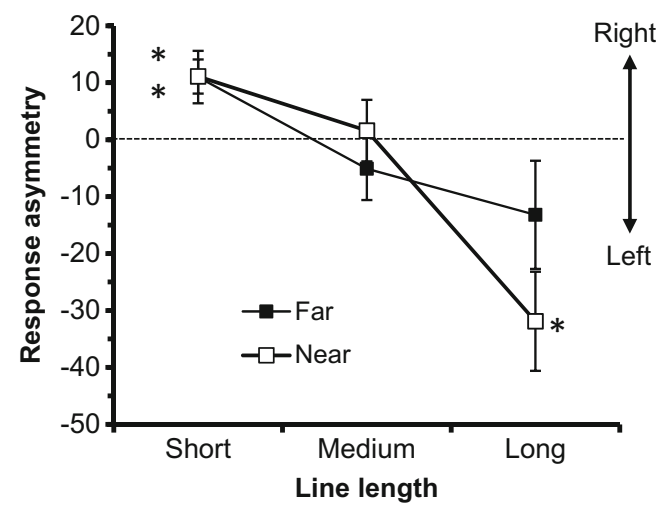

Fig. 2 Graph showing the mean response asymmetry scores for horizontal lines in Experiment 1. The data show the interaction between viewing distance and stimulus length. Error bars show $\pm S E$ s of the means. Conditions that are significantly different from zero (no bias) are marked with an asterisk pathway may be particularly important in landmark line bisection tasks and pseudoneglect (Foxe, McCourt \& Javitt, 2003; Waberski et al., 2008). In contrast, there was no effect of viewing distance for the short and medium lines. An explanation for this dissociation between line lengths will be developed in the General Discussion.

\section{Experiment 2}

In the second experiment, we adapted the techniques used in Experiment 1 to investigate the effects of stimulus length and viewing distance in the vertical dimension. The stimuli and procedure used in this experiment were exactly the same as those in the first experiment. The second experiment therefore provided an opportunity to test whether the effects observed in Experiment 1 are a general property of changes in stimulus length and viewing distance, or whether they relate specifically to mechanisms associated with the processing of horizontal lines-and by implication, the left and right cerebral hemispheres.

As with the horizontal axis, there are also asymmetries in attention along the vertical axis. These asymmetries are reported for patients with neglect (Halligan \& Marshall, 1993; 1994) and for the general population (Bradshaw, Nettleton, Nathan, \& Wilson, 1985; Drain \& Reuter-Lorenz, 1996; McCourt \& Olafson, 1997; Nicholls et al., 2004; van Vugt, Fransen, Creten, \& Paquier, 2000). For both groups, there is a bias toward bisecting lines above the true center.

Upward biases for line bisection have been explained with reference to the relative activations of the dorsal and ventral streams, which are connected to the lower and upper visual fields, respectively (Drain \& Reuter-Lorenz, 1996; Previc, 1990). The dorsal stream is thought to process where a stimulus is located, whereas the ventral stream is specialized for processing what the stimulus is (Goodale \& Milner, 1992). Therefore, the act of line bisection, which favors the "what" mechanism, may cause more activation of the ventral processing stream, which causes a bias of attention toward the upper hemispace. In this respect, this model is quite similar to the activation model proposed by Kinsbourne (1993) for the horizontal axis - except that differential activation of the visual streams rather than the hemispheres drives the asymmetry.

In addition to the horizontal dimension, Heber et al. (2010) investigated the interaction between line length and viewing distance for the vertical dimension. For the greyscales task, they found that the upward attentional bias was stronger in near than in far space. Thus, vertical stimuli appear to be affected by changes in viewing distance in a similar fashion to horizontal stimuli (McCourt \& Jewell, 1999). Heber et al. also observed an effect of stimulus length for the greyscales task, whereby the upward bias was stronger for longer stimuli. The factors of viewing distance and length did not interact for 
the greyscales task. Heber et al. also administered a vertical version of the landmark task and, contrary to what might be predicted, found no significant effect of length or viewing distance.

The experimental design used in Experiment 2 was identical to that used in Experiment 1-except that the display was simply rotated by $90^{\circ}$ to produce vertical lines. The following predictions were made for short, medium, and long lines.

(a) The horizontal short lines produced a reliable crossover effect in Experiment 1. To our knowledge, no research has investigated whether a crossover effect can also be observed for vertical lines. If this effect is simply related to having a very short line, a crossover effect should be observed. Conversely, if no crossover effect were found, it would suggest that the effect is related to something specific to the processing of left and right along the horizontal axis.

Another issue to be considered is how any asymmetry for short lines is affected by viewing distance. Heber et al. (2010) demonstrated that the upward bias for the greyscales task was stronger in near than in far space for relatively long stimuli. If this effect of viewing distance generalizes to short stimuli, a similar effect should be observed for very short lines.

(b) The medium lines produced no response asymmetry in Experiment 1. If the vertical and horizontal alignments behave in the same way, a similar null effect would be predicted in Experiment 2. Like the short lines, if the effect of viewing distance expected for long lines generalized to medium lines, an effect of viewing distance would be expected.

(c) The long lines were expected to yield an upward response bias consistent with other research in the area (Bradshaw et al., 1985; Drain \& Reuter-Lorenz, 1996; McCourt \& Olafson, 1997; Nicholls et al., 2004; van Vugt et al., 2000). In line with the effect of distance reported by Heber et al. (2010), the upward bias was expected to be stronger for lines in near than in far space.

\section{Method}

Participants Nineteen university students participated in this experiment. One participant was removed for clearly not understanding the task and achieving an error score of $72 \%$. The remaining 18 students (13 females, five males) had ages ranging between 18 and 26 years ( $M=19.50$ years). All participants were right-handed according to the FLANDERS handedness survey (range +5 to $+10, M=+9.22$ ). All other characteristics were the same as in Experiment 1.
Apparatus, stimuli, and procedure All aspects of the method used in Experiment 2 were identical to those of Experiment 1 , with the following exceptions. First, the stimuli were presented vertically rather than horizontally. To accommodate this change, the monitor was simply rotated by $90^{\circ}$ in a clockwise direction. It was necessary to rotate the monitor rather than redraw the stimuli because of the very long lines used in the "far long-line" condition, which would not fit on the screen otherwise. In relation to the response, participants were asked to indicate whether the upper or the lower line segment appeared longer. To provide a natural mapping between response and stimulus location, the keyboard was also rotated by $90^{\circ}$. Participants pressed the " $\mathrm{j}$ " key to indicate that the upper segment was longer, and the " $\mathrm{f}$ " key to indicate that the lower segment was longer. The responses were made with the index fingers of the left and right hands, and the hand of response was balanced between participants. Participants completed one block of 12 practice trials prior to commencing the experimental trials.

\section{Results and discussion}

A measure of error was calculated using the method described in Experiment 1. The average overall error rate was $41.98 \%(S D=6.9)$. An independent-samples $t$ test demonstrated that the error rate was significantly higher for the vertical than for the horizontal condition $[t(36)=7.163, p<.001]$. The increased difficulty for the vertical trials may reflect the operation of a specialized mechanism for symmetry detection along the horizontal axis, which makes horizontal trials easier (Wenderoth, 1994).

The response asymmetry was calculated using a procedure similar to that described in Experiment 1. In this case, however, "upper longer" responses were subtracted from "lower longer" responses. Negative scores therefore indicated a bias toward perceiving the upper segment of the lines as longer. A series of one-sample $t$ tests revealed no significant differences from zero (i.e., no response asymmetry) for the very short lines in either the near or the far condition. It is therefore apparent that the crossover effect is limited to horizontal lines and does not occur in the vertical dimension. The implications for this dissociation will be outlined in the General Discussion. For the medium lines, there was also no significant asymmetry toward either "upper" or "lower" responses. This null effect is consistent with Experiment 1 and may indicate that lines of moderate length do not elicit an attentional asymmetry. Finally, for the long lines, we observed an asymmetry for the near condition $[t(17)=2.488, p=.023]$, which is in accord with the upward bias reported by many other researchers (Bradshaw et al., 1985; Drain \& Reuter-Lorenz, 1996; McCourt \& Olafson, 1997; Nicholls et al., 2004; van Vugt et al., 2000). In contrast, no significant upward bias for 
long lines emerged in the far condition, and this may reflect the effect of viewing distance reported by Heber et al. (2010).

The response asymmetry data were then analyzed using the ANOVA described for Experiment 1. We found a significant effect of length $\left[F(2,34)=4.362, p=.021, \eta_{\mathrm{p}}{ }^{2}=.204\right]$. Posthoc analyses (Bonferroni corrected) revealed no significant difference between the short and medium lines $[t(17)=$ $0.064, p=.950]$ or between the short and long lines $[t(17)=$ $2.189, p=.042]$. In contrast, there was a significant difference between the medium and long lines $[t(17)=3.089, p=.007]$. It therefore appears that length did affect the response asymmetry - but that this effect was confined to the long lines. The lack of a difference between the short and medium lines most likely reflects the fact that a crossover effect did not occur. No effect of distance emerged $\left[F(1,17)=0.022, p=.883, \eta_{\mathrm{p}}{ }^{2}=\right.$ $.001]$. Despite the fact that the one-sample $t$ tests detected a difference in the biases for long lines, but not for medium or short lines, the interaction between viewing distance and stimulus length failed to reach statistical significance $[F(2,34)=$ $1.374, p=.267, \eta_{\mathrm{p}}{ }^{2}=.075$ ] (see Fig. 3).

Unlike the first experiment, Experiment 2 failed to demonstrate an interaction between stimulus length and viewing distance. That said, the patterns of results are remarkably similar between the experiments. To test this explicitly, an omnibus ANOVA was carried out on all of the data, with Experiment $(1,2)$ as a between-participants factor. The ANOVA produced a strong effect of length $\left[F(2,74)=14.772, p<.001, \eta_{\mathrm{p}}{ }^{2}=\right.$ .285]. We also observed a trend for length to interact with experiment $\left[F(2,74)=2.740, p=.071, \eta_{\mathrm{p}}{ }^{2}=.069\right]$, most likely reflecting the fact that a crossover effect was observed in Experiment 1, but not Experiment 2. There was no effect of viewing distance $[F(1,37)=0.798, p=.377]$, but a strong interaction was apparent between viewing distance and length $\left[F(2,74)=7.104, p=.002, \eta_{\mathrm{p}}^{2}=.161\right]$. Importantly, this interaction was not affected by experiment $[F(2,74)=$

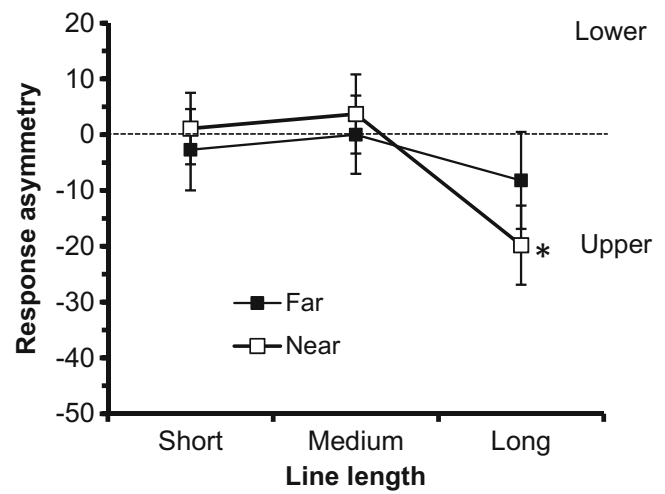

Fig. 3 Graph showing the mean response asymmetry scores for vertical lines in Experiment 2. The data show the interaction between viewing distance and stimulus length. Error bars show $\pm S E$ s of the means. The condition that is significantly different from zero (no bias) is marked with an asterisk
$0.378, p=.687]$. This lack of an interaction supports the proposition that both experiments showed no effect of viewing distance for short and medium lines, whereas the attentional bias was stronger in the near than in the far condition for long lines.

\section{General discussion}

In Experiment 1, we examined the effects of stimulus length and viewing distance on attentional asymmetries along the horizontal axis for a landmark task. In accord with previous research, the leftward bias for long lines (pseudoneglect) reversed to a rightward bias for very short lines (the crossover effect; McCourt \& Jewell, 1999; Rueckert et al., 2002). In Experiment 2, we used exactly the same procedure as in the first experiment-except that the screen was rotated $90^{\circ}$ to form vertical lines. In this case, the expected upward bias was found for long lines (Bradshaw et al., 1985; Drain \& Reuter-Lorenz, 1996; McCourt \& Olafson, 1997; Nicholls et al., 2004; van Vugt et al., 2000). There was, however, no reversal of this response asymmetry for the very short lines. The data therefore demonstrate that the crossover effect is not a general property of short lines, and pertains specifically to horizontal lines.

Although it seems reasonable to suggest that the dissociation in the crossover effect between the horizontal and vertical axes is related to differences in cognitive/neural mechanisms, another possibility cannot be completely ruled out at this stage. It is known that asymmetries exist in judgments of length in the vertical and horizontal dimensions. This asymmetry is clearly demonstrated by the horizontal vertical illusion (Avery \& Day, 1969), in which the length of the vertical segment of an inverted " $T$ " is overestimated relative to the horizontal segment. This overestimation is thought to arise from a vertical bias (in which the length of vertical lines is overestimated) and a bisection bias (in which bisected lines appear shorter) (Josev, Forte \& Nicholls, 2011). The vertical bias is more relevant to the present study and results in an overestimation of length of 5\% in vertical as compared to horizontal lines (Charras \& Lupiáñez, 2010). Bearing this in mind, it is possible that participants perceived the vertical line to be slightly longer than the horizontal line. Given that the shortness of the line is of paramount importance to the generation of the crossover effect, it is therefore possible that the vertical lines failed to produce a crossover effect because they were not short enough. That said, the difference in perceived length was likely very small. A 5\% increase in the length of the shortest vertical line would increase the perceived visual angle from $1.27^{\circ}$ to $1.33^{\circ}$. This slightly larger perceived visual angle is still well within the range of stimulus lengths reported to produce a crossover effect in the general population (Rueckert et al., 2002). We therefore believe that the failure 
to observe a crossover effect for vertical trials is not related to an overestimation of length in the vertical dimension.

The finding that the crossover effect is specific to the horizontal axis is relevant to models that attempt to explain the effect within the general population. For example, Mennemeier et al. (2005) suggested that crossover occurs because of the interplay between two separate processes: (1) magnitude estimation and (2) orientation bias. Magnitude estimation refers to a phenomenon whereby the length of short lines is systematically overestimated, whereas the length of long lines is underestimated (Tegner \& Levander, 1991). Orientation bias refers to a rightward shift of attention caused by a stronger inhibitory influence of the left hemisphere. Whereas Kinsbourne (1970) hypothesized a dominant role for the left hemisphere and a rightward attentional bias in the general population, it should be noted that most of the literature reviewed in the introduction of this article suggests the opposite - right hemisphere dominance for spatial attention and a leftward attentional bias. Notwithstanding this point, Mennemeier et al. (2005) suggested that once attention is directed to the right, overestimation of the length of short lines will cause the bisection mark to fall short of the true center (rightward bias). Conversely, for long lines, underestimation of length will cause the bisection mark to be placed past the true center (leftward bias).

The upshot of the theory proposed by Mennemeier et al. (2005) is that short lines have the effect of reversing the preexisting attentional bias, whereas long lines exacerbate the existing bias. If this were the case, however, one would expect a crossover effect for the vertical dimension. Specifically, the bias of attention toward the upper hemispace caused by activation of the ventral system (Drain \& ReuterLorenz, 1996; Previc, 1990) should be reversed to a bias toward the lower hemispace by the overestimation of length for very short lines. Figure 3 clearly shows no evidence of a downward bias for the very short lines-contradicting the theory proposed by Mennemeier et al. (2005).

It therefore appears that a mechanism pertaining specifically to left-right asymmetries needs to be incorporated into a model of the crossover effect in the general population. One such model was developed by Rueckert et al. (2002). Although Rueckert's model also refers to attentional biases and an under-/overestimation of line length, the model was not discussed by Mennemeier et al. (2005)_ despite the fact that the results of that study were reviewed. The model proposed by Rueckert et al. refers to a leftward attentional bias generated by spatial attention mechanisms located in the right hemisphere. This leftward attentional bias underlies most theories of pseudoneglect (Bultitude \& Aimola-Davies, 2006; Nicholls \& Roberts, 2002) and the neural/cognitive models that underlie them (Siman-Tov et al., 2007; Thiebaut de Schotten et al., 2011). Rueckert et al. (2002) also discussed the relative under- and overestimations of lines of different lengths. In this case, however, cerebral asymmetries for the processing of line length were also discussed. Tsal and Shalev (1996) demonstrated that the length of short lines was overestimated more in the right visual field, whereas Masin and Sartori (1988) demonstrated that the length of long lines was overestimated on the left. Combined, these asymmetrical length estimation effects can account for the slight rightward bias observed for very short lines and the larger leftward bias for longer lines. Because the mechanism includes asymmetries between the hemispheres in their estimations of relative length, the model can explain why crossover only occurs for the horizontal dimension.

An alternative model has been proposed by Benwell et al. (2013). In this case, they argued that long lines may load more heavily on right hemisphere resources because they need to incorporate bilateral peripheral input, or a larger spotlight of attention. Alternatively, the researchers suggested an extinction-like process in which the larger extension of long lines into the periphery exacerbates the attentional imbalance. Such attentional accounts draw on Anderson's (1996) mathematical model of line bisection in neglect patients, which described the salience of lines as a function of their spatial position. The model developed by Anderson provides a unitary mathematical model to describe rightward bisection in neglect patients as well as the leftward bias for very short lines (crossover effect). That said, Anderson's model does not refer to pseudoneglect and the crossover effect in a healthy population. It is, therefore, still difficult to know how the reduced engagement of the right hemisphere discussed by Benwell et al. (2013) would actually lead to left hemisphere activation and a rightward bias for line bisection (i.e., the crossover effect) - like that observed in the present study.

The present set of experiments also identified an interaction between viewing distance and stimulus length. The expected effect of viewing distance, in which the response bias is attenuated for far viewing conditions, was found only for long lines. No effect of viewing distance was observed for short or medium lines. The pattern of interaction was found for horizontal lines and also occurred for vertical lines - albeit just failing to reach statistical significance. An omnibus ANOVA revealed no difference in the patterns of interaction between the horizontal and vertical dimensions. It is therefore evident that this effect is a general property of line length and is processed in the same way along the horizontal and vertical axes. Such a proposition fits with $\mathrm{fMRI}$ research carried out by Fink, Marshall, Weiss, and Zilles (2001). They measured brain activation during horizontal and vertical landmark tasks and found the same activation of the inferior parietal cortex irrespective of orientation.

Within the context of the present experiments, one can only speculate about the cognitive/neural mechanisms that might underlie the interaction between stimulus length and viewing distance. One possibility is that short and long lines favor 
different processing strategies, which may differentially engage the cerebral hemispheres. For example, long lines are more likely to engage a global processing strategy, as well as visual processing associated with low spatial frequencies. Both global processing (Poynter \& Roberts, 2012; Van Kleeck, 1989) and the processing of low spatial frequencies (Sergent, 1982) are suited to the processing style of the right hemisphere. In contrast, short and medium lines may favor local/high-frequency processing, which load more heavily on left hemisphere processing (Sergent, 1982; Van Kleeck, 1989). The processing of long lines within the right hemisphere may favor differential activation of the ventral and dorsal streams within that hemisphere, which are specialized for processing far and near stimuli (respectively). Although both hemispheres contain ventral and dorsal visual pathways, the interaction between these pathways may be especially important. As we noted in the introduction, Thiebaut de Schotten et al. (2011) found that the middle superior longitudinal fasciculus in the right hemisphere plays a particularly important role in communication between the ventral and dorsal systems. In addition, clinical research has shown that dissociations in neglect between near and far space are restricted to lesions in the right hemisphere (Halligan \& Marshall, 1991; Vuilleumier, Valenza, Mayer, Reverdin, \& Landis, 1998). It is therefore possible that a dissociation between ventral (far) and dorsal (near) processing occurs only when the stimuli are suited to a right hemisphere style of processing. Although this mechanism relates to a cerebral asymmetry, it would apply equally to vertical and horizontal lines. Although no specific evidence supports this proposal, research by Szpak, Thomas, and Nicholls (2016) has demonstrated that the distal bias is strongest for radial lines when they are presented to the left hemispace. Given that the distal dimension mapped onto the upper visual field in their study, it would be interesting to see whether the upward bias would be stronger for vertical lines presented in the left hemispace. Such an effect would be consistent with the proposal that the right hemisphere plays a dominant role in ventral-dorsal dissociations.

In summary, the present set of experiments both point toward the importance of right hemisphere processing in effects related to line bisection. In relation to the crossover effect, cerebral asymmetries in the under-/overestimation of line length may give rise to the rightward bias observed in the general population. In relation to the effect of viewing distance, the interaction between the ventral and dorsal pathways within the right hemisphere may give rise to the dissociation observed for long lines.

Author note This study was supported with funding from an ARC Discovery Project grant (No. DP130100541). Thanks are extended to Lauren Nicholls for drawing Fig. 1, and to the reviewers for their feedback on the manuscript.

\section{References}

Aimola, L., Schindler, I., Simone, A., \& Venneri, A. (2012). Near and far space neglect: Task sensitivity and anatomical substrates. Neuropsychologia, 50, 1115-1123.

Anderson, B. (1996). A mathematical model of line bisection behaviour in neglect. Brain, 119, 841-850.

Avery, G. C., \& Day, R. H. (1969). Basis of the horizontal-vertical illusion. Journal of Experimental Psychology, 81, 376-380. doi:10. 1037/h0027737

Benwell, C. S. Y., Harvey, M., Gardner, S., \& Thut, G. (2013). Stimulusand state-dependence of systematic bias in spatial attention: Additive effects of stimulus-size and time-on-task. Cortex, 49, 827-836.

Benwell, C. S. Y., Harvey, M., \& Thut, G. (2014). On the neural origin of pseudoneglect: EEG-correlates of shifts in line bisection performance with manipulation of line length. NeuroImage, 86, 370380. doi:10.1016/j.neuroimage.2013.10.014

Benwell, C. S. Y., Thut, G., Grant, A., \& Harvey, M. (2014). A rightward shift in the visuospatial attention vector with healthy aging. Frontiers in Ageing Neuroscience, 6(113), 1-11. doi:10.3389/ fnagi.2014.00113

Berti, A., \& Frassinetti, F. (2000). When far becomes near: Remapping of space by tool use. Journal of Cognitive Neuroscience, 12, 415-420.

Bjoertomt, O., Cowey, A., \& Walsh, V. (2002). Spatial neglect in near and far space investigated by repetitive transcranial magnetic stimulation. Brain, 125, 2012-2022.

Bowers, D., \& Heilman, K. M. (1980). Pseudoneglect: Effects of hemispace on a tactile line bisection task. Neuropsychologia, 18, 491-498. doi:10.1016/0028-3932(80)90151-7

Bradshaw, J. L., Nettleton, N. C., Nathan, G., \& Wilson, L. (1985). Bisecting rods and lines: Effects of horizontal and vertical posture on left-side underestimation by normal subjects. Neuropsychologia, 23, 421-425.

Bultitude, J., \& Aimola-Davies, A. M. (2006). Putting attention on the line: Investigating the activation-orientation hypothesis of pseudoneglect. Neuropsychologia, 44, 1849-1858.

Charras, P., \& Lupiáñez, J. (2010). Length perception of horizontal and vertical bisected lines. Psychological Research, 74, 196-206.

Chokron, S., \& Imbert, M. (1993). Influence of reading habits on line bisection. Cognitive Brain Research, 1, 219-222.

Drain, M., \& Reuter-Lorenz, P. A. (1996). Vertical orienting control: Evidence for attentional bias and "neglect" in the intact brain. Journal of Experimental Psychology: General, 125, 139-158. doi: 10.1037/0096-3445.125.2.139

Fink, G. R., Marshall, J. C., Shah, N. J., Weiss, P. H., Halligan, P. W., Grosse-Ruyken, M., . . . Freund, H. J. (2000). Line bisection judgments implicate right parietal cortex and cerebellum as assessed by fMRI. Neurology, 54, 1324-1331.

Fink, G. R., Marshall, J. C., Weiss, P. H., \& Zilles, K. (2001). The neural basis of vertical and horizontal line bisection judgments: An fMRI study of normal volunteers. NeuroImage, 14, s59-s67.

Foxe, J. J., McCourt, M. E., \& Javitt, D. C. (2003). Parietal control of visuospatial attention: Line bisection judgments evaluated with high-density electrical mapping and source analysis. NeuroImage, 19, 710-726.

Gamberini, L., Seraglia, B., \& Priftis, K. (2008). Processing of peripersonal and extrapersonal space using tools: Evidence from visual line bisection in real and virtual environments. Neuropsychologia, 46, 1298-12304.

Goodale, M. A., \& Milner, A. D. (1992). Separate visual pathways for perception and action. Trends in Neurosciences, 15, 20-25. doi:10. 1016/0166-2236(92)90344-8 
Halligan, P. W., \& Marshall, J. C. (1991). Left neglect for near but not far space in man. Nature, 350, 498-500.

Halligan, P. W., \& Marshall, J. C. (1993). The bisection of horizontal and radial lines: A case study of normal controls and ten patients with left visuospatial neglect. International Journal of Neuroscience, 70, 149-167.

Halligan, P. W., \& Marshall, J. C. (1994). Focal and global attention modulate the expression of visuo-spatial neglect: A case study. Neuropsychologia, 32, 13-21.

Heber, I. A., Siebertz, S., Wolter, M., Kuhlen, T., \& Fimm, B. (2010). Horizontal and vertical pseudoneglect in peri- and extrapersonal space. Brain and Cognition, 73, 160-166.

Heilman, K. M., \& Van Den Abell, T. (1980). Right-hemisphere dominance for attention: The mechanisms underlying hemispheric asymmetries of inattention (neglect). Neurology, 30, 327-330.

Heilman, K. M., Watson, R. T., \& Valenstein, E. (1993). Neglect and related disorders. In K. M. Heilman \& E. Valenstein (Eds.), Clinical neuropsychology (pp. 279-336). New York, NY: Oxford University Press.

Iriki, A., Tanaka, M., \& Iwamura, Y. (1996). Coding of modified body schema during tool use by macaque postcentral neurones. NeuroReport, 7, 2325-2330.

Jewell, G., \& McCourt, M. E. (2000). Pseudoneglect: A review and metaanalysis of performance factors in line bisection tasks. Neuropsychologia, 38, 93-110. doi:10.1016/S0028-3932(99) 00045-7

Josev, E. K., Forte, J. D., \& Nicholls, M. E. R. (2011). Left of centre: Asymmetries for the horizontal vertical line illusion. Psychological Research, 75, 435-443.

Kinsbourne, M. (1970). The cerebral basis of lateral asymmetries in attention. Acta Psychologica, 33, 193-201.

Kinsbourne, M. (1993). Orientational bias model of unilateral neglect: Evidence from attentional gradients within hemispace. In I. H. Robertson \& J. C. Marshall (Eds.), Unilateral neglect: Clinical and experimental studies (pp. 63-86). Hillsdale, NJ: Erlbaum.

Konen, C. S., \& Kastner, S. (2008). Two hierarchically organised neural structures for object information in human visual cortex. Nature Neuroscience, 11, 224-231.

Loftus, A. M., \& Nicholls, M. E. R. (2012). Testing the activation-orientation account of spatial attentional asymmetries using transcranial direct current stimulation. Neuropsychologia, 50, 2573-2576. doi: 10.1016/j.neuropsychologia.2012.07.003

Loftus, A., Nicholls, M. E. R., Mattingley, J. B., Chapman, H. L., \& Bradshaw, J. L. (2009). Pseudoneglect for the bisection of mental number lines. Quarterly Journal of Experimental Psychology, 62, 925-945

Longo, M. R., \& Lourenco, S. F. (2006). On the nature of near space: Effects of tool use and the transition to far space. Neuropsychologia, 44, 977-981

Longo, M. R., Trippier, S., Vagnoni, E., \& Lourenco, S. F. (2015). Right hemisphere control of visuospatial attention in near space. Neuropsychologia, 70, 350-357. doi:10.1016/j.neuropsychologia. 2014.10.035

Masin, S. C., \& Sartori, G. (1988). An experimental study of halfmeridional differences. Psychological Research, 50, 33-42.

McCourt, M. E. (2001). Performance consistency of normal observers in forced-choice tachistoscopic visual line bisection. Neuropsychologia, 39, 1065-1076.

McCourt, M. E., \& Garlinghouse, M. (2000). Asymmetries of visuospatial attention are modulated by viewing distance and visual field elevation: Pseudoneglect in peripersonal and extrapersonal space. Cortex, 36, 715-731.

McCourt, M. E., \& Jewell, G. (1999). Visuospatial attention in line bisection: Stimulus modulation of pseudoneglect. Neuropsychologia, $37,843-855$.
McCourt, M. E., \& Olafson, C. (1997). Cognitive and perceptual influences on visual line bisection: Psychophysical and chronometric analyses of pseudoneglect. Neuropsychologia, 35, 369-380.

Mennemeier, M., Pierce, C. A., Chatterjee, A., Anderson, B., Jewell, G., Dowler, R., . . . Mark, V. W. (2005). Biases in attentional orientation and magnitude estimation explain crossover: Neglect is a disorder of both. Journal of Cognitive Neuroscience, 17, 1194-1211. doi:10. $1162 / 0898929055002454$

Moonaghan, P., \& Shillcock, R. (1998). The cross-over effect in unilateral neglect: Modelling detailed data in the line-bisection task. Brain, 121, 907-921.

Nicholls, M. E. R., \& Roberts, G. R. (2002). Pseudoneglect: A scanning, pre-motor or attentional bias? Cortex, 38, 113-136.

Nicholls, M. E. R., Bradshaw, J. L., \& Mattingley, J. B. (1999). Freeviewing perceptual asymmetries for the judgement of shade, numerosity and size. Neuropsychologia, 37, 307-314.

Nicholls, M. E. R., Mattingley, J. B., Berberovic, N., Smith, A., \& Bradshaw, J. L. (2004). An investigation of the relationship between free-viewing perceptual asymmetries for vertical and horizontal stimuli. Cognitive Brain Research, 19, 289-301.

Nicholls, M. E. R., Thomas, N. A., Loetscher, T., \& Grimshaw, G. M. (2013). The Flinders Handedness survey (FLANDERS): A brief measure of skilled hand preference. Cortex, 49, 2914-2926.

Nicholls, M. E. R., Roden, S., Thomas, N. A., Loetscher, T., Spence, C. J., \& Forte, J. D. (2014). Close to me: Asymmetrical environments do not affect spatial attention. Ergonomics, 57, 876-885.

Nicholls, M. E. R., Jones, C. A., \& Robertson, J. S. (2016). Heading to the right: The effect of aperture width on navigation asymmetries for miniature remote-controlled vehicles. Journal of Experimental Psychology: Applied. Advance Online Publication. doi:10.1037/ xap0000078

Poynter, W., \& Roberts, C. (2012). Hemispheric asymmetries in visual search. Laterality, 17, 711-726.

Previc, F. H. (1990). Functional specialization in the lower and upper visual fields in humans: Its ecological origins and neurophysiological implications. Behavioral and Brain Sciences, 13, 519-542.

Rinaldi, L., Di Luca, S., Henik, A., \& Girelli, L. (2014). Reading direction shifts visuospatial attention: An Interactive Account of attentional biases. Acta Psychologica, 151, 98-105. doi:10.1016/j.actpsy. 2014.05.018

Rizzolatti, G., Matelli, M., \& Pavesi, G. (1983). Deficits in attention and movement following the removal of postarcuate (area 6) and prearcuate (area 8) cortex in macaque monkeys. Brain, 106, 655673.

Rueckert, L., Deravanesian, A., Baboorian, D., Lacalamita, A., \& Repplinger, M. (2002). Pseudoneglect and the cross-over effect. Neuropsychologia, 40, 162-173.

Sergent, J. (1982). The cerebral balance of power: Confrontation or cooperation? Journal of Experimental Psychology: Human Perception and Performance, 8, 253-272. doi:10.1037/0096-1523.8.2.253

Siman-Tov, T., Mendelsohn, A., Schonberg, T., Avidan, G., Podlipsky, I., Pessoa, L., . . . Hendler, T. (2007). Bihemispheric leftward bias in a visuospatial attention-related network. Journal of Neuroscience, 27, 11271-11278.

Szpak, A., Thomas, N. A., \& Nicholls, M. E. R. (2016). Hemispheric asymmetries in perceived depth revealed through a radial line bisection task. Experimental Brain Research, 234, 807-813.

Tegner, R., \& Levander, M. (1991). The influence of stimulus properties on visual neglect. Journal of Neurology, Neurosurgery, and Psychiatry, 54, 882-887.

Thiebaut de Schotten, M., Dell'Acqua, F., Forkel, S. J., Simmons, A., Vergani, F., Murphy, D. G. M., \& Catani, M. (2011). A lateralized brain network for visuospatial attention. Nature Neuroscience, 14, 1245-1246. doi:10.1038/nn.2905 
Thomas, N. A., Castine, B. R., Loetscher, T., \& Nicholls, M. E. R. (2015). Upper visual field distractors preferentially bias attention to the left. Cortex, 64, 179-193.

Tsal, Y., \& Shalev, L. (1996). Inattention magnifies perceived length: The attentional receptive field hypothesis. Journal of Experimental Psychology: Human Perception and Performance, 22, 233-243. doi:10.1037/0096-1523.22.1.233

Van Kleeck, M. H. (1989). Hemispheric differences in global versus local processing of hierarchical visual stimuli by normal subjects: New data and a meta-analysis of previous studies. Neuropsychologia, 27, $1165-1178$.

van Vugt, P., Fransen, I., Creten, W., \& Paquier, P. (2000). Line bisection performances of 650 normal children. Neuropsychologia, 38, 886895.

Varnava, A., McCarthy, M., \& Beaumont, J. G. (2002). Line bisection in normal adults: Direction of attentional bias for near and far space. Neuropsychologia, 40, 1372-1378.
Vuilleumier, P., Valenza, N., Mayer, E., Reverdin, A., \& Landis, T. (1998). Near and far visual space in unilateral neglect. Annals of Neurology, 43, 406-410.

Waberski, T. D., Gobbelé, R., Lamberty, K., Buchner, H., Marshall, J. C., \& Fink, G. R. (2008). Timing of visuo-spatial information processing: Electrical source imaging related to line bisection judgements. Neuropsychologia, 46, 1201-1210. doi:10.1016/j. neuropsychologia.2007.10.024

Weiss, P. H., Marshall, J. C., Wunderlich, G., Tellmann, L., Halligan, P. W., Freund, H., . . . Fink, G. R. (2000). Neural consequences of acting in near versus far space: A physiological basis for clinical dissociations. Brain, 123, 2531-2541.

Wenderoth, P. (1994). The salience of vertical symmetry. Perception, 23, 221-236.

Wilkinson, D., \& Halligan, P. (2003). The effect of stimulus size on bisection judgements in near and far space. Visual Cognition, 10, 319-340. 